\title{
Satisfação de Clientes em Serviços de Educação Física Supervisionada na Região de Curitiba, Brasil: Aplicação do QSCSEF
}

\author{
Customer Satisfaction in Supervised Physical Education Services in Curitiba, \\ Brazil: Application of QSCSEF
}

Jhomyr Dias Modesto ${ }^{1}$, Leandra Ulbricht ${ }^{1}$, Eduardo Borba Neves ${ }^{1,2^{*}}$

ARTIGO ORIGINAL | ORIGINAL ARTICLE

\begin{abstract}
O presente estudo objetivou avaliar a satisfação de clientes de serviços em Educação Física supervisionada em Curitiba e Região Metropolitana. Foi realizado um estudo exploratório descritivo com 921 indivíduos, sendo 436 do sexo masculino e 485 do sexo feminino, com média de idade de $26.9 \pm 9.4$ anos. O instrumento de pesquisa utilizado foi o Questionário de Satisfação de Clientes em Serviços de Educação Física. Como principais resultados, verificou-se que dentre os serviços avaliados, as academias de musculação se apresentaram como o serviço mais utilizado (61.9\%), as academias de dança apresentaram maior qualidade e satisfação e a média de tempo de utilização dos serviços foi de $25.2 \pm 33.1$ meses, sendo as escolas de esportes coletivos as que apresentaram as maiores médias. Concluiu-se que as academias de musculação se caracterizaram como o serviço mais utilizado, as academias de yôga/pilates com o maior percentual de problemas relatados e as academias de dança o maior índice de qualidade de serviço e satisfação dos clientes.

Palavras-chaves: Educação Física e Treinamento, Satisfação dos Consumidores, Mercado de Trabalho.
\end{abstract}

\begin{abstract}
This study aimed to assess the client satisfaction with supervised services in physical education in Curitiba Metropolitan Region. A descriptive exploratory study with 921 subjects, 436 males and 485 females, with a mean age of $26.9 \pm 9.4$ years was conducted. The research instrument used was the Client Satisfaction Questionnaire in Physical Education Services. As main results, it was found that among the evaluated services, fitness gyms were found to be the most used service $(61.9 \%)$, dance academies had higher quality and satisfaction and the average time of use of services was $25.2 \pm 33.1$ months, being the team sports schools those with the highest averages. It was concluded that the bodybuilding gyms have been characterized as the most used service academies, yoga/pilates academies presented the highest percentage of problems reported and dance academies achieved the highest quality of service and customer satisfaction. Keywords: Physical Education and Training, Consumer Satisfaction, Job Market.
\end{abstract}

\footnotetext{
Artigo recebido a 30.04.2015; Aceite a 01.08.2015

${ }^{1}$ Programa de Pós-Graduação em Engenharia Biomédica, Universidade Tecnológica Federal do Paraná -UTFPR, Curitiba, Brasil.

${ }^{2}$ Instituto de Pesquisa da Capacitação Física do Exército - IPCFEX, Rio de Janeiro, Brasil.

* Autor correspondente: Av João Luis Alves s/nº Fortaleza de São João - Urca, 22291-090, Rio de Janeiro, Brasil. E-mail: borbaneves@hotmail.com
} 


\section{INTRODUÇÃO}

O sucesso de uma empresa depende de sua capacidade de prever as tendências e antecipar o futuro (Fernandes, 2013), e sua competitividade se fundamenta na capacidade de perceber as mudanças e preparar-se para enfrentá-las (Costa, Mansur, Freitas, \& Carvalho, 2007; Fernandes, 2013). A crescente competitividade no mercado, o elevado nível de exigência dos clientes e a busca pela redução de custos faz com que as empresas dependam cada vez mais de mecanismos que garantam eficiência na administração e na gestão da qualidade (Saldanha, 2013). A qualidade pode ser compreendida como o atendimento total das necessidades e expectativas, extrínsecas e intrínsecas, do cliente (Saldanha, 2013) e está diretamente relacionada com a fidelização do mesmo aos produtos ou serviços oferecidos pela empresa (Larán \& Espinoza, 2004).

Uma importante característica relacionada à fidelização é a análise do comportamento do cliente, que é influenciado por fatores culturais, sociais, pessoais e psicológicos (Fernandes, 2013). Dentro desses fatores, destaca-se o grau de satisfação, que é definido como o julgamento formado durante o uso ou consumo de produto ou serviço, constituindo uma reação ou sentimento em relação a uma expectativa (Batista, Couto, Botelho, \& Faias, 2014; Borrego, Leitão, Alves, Silva, \& Palmi, 2000; Gabriel, Pelissari, \& Oliveira, 2014; Larán \& Espinoza, 2004; Rech, Fermino, Hallal, \& Reis, 2011; Zequinão \& Cardoso, 2013).

No contexto da Educação Física encontramos o mercado do fitness, que está cada vez mais crescente, apresentando um aumento de $61 \%$ no número de academias ao redor do mundo e crescimento de receita de $55 \%$ entre os anos de 2005 e 2011 (Menezes, 2013). Valores elevados também podem ser vistos no Brasil, que já se caracteriza como o segundo país mundial em número de academias, ficando atrás somente dos Estados Unidos (Menezes, 2013). Este crescimento aumentou a competição entre as empresas, muitas vezes pelo mesmo consumidor, gerando a premissa de compreensão das necessidades e anseios dos clientes, a fim de garantir sua satisfação e permanência (Medeiros, Andrade, Oliveira, Júnior, \& Sousa, 2012).
Além disso, sabe-se que a percepção de satisfação é um importante mediador da motivação para a prática de atividade física, e contribui expressivamente para a adoção do comportamento fisicamente ativo, uma vez que indivíduos com maior satisfação decorrente da prática de atividade física são mais ativos fisicamente (Ferreira, 2012; Rech et al., 2011). Tal característica também pode ser visualizada em atletas onde estudos têm apontado que a satisfação relaciona-se positivamente com o desempenho e negativamente com a desistência ou abandono da modalidade (Borrego et al., 2000; Gomes, Pereira, \& Pinheiro, 2008).

Apesar da importância do tema, poucas organizações dentro da Educação física se preocupam em avaliar seus serviços ou obter um feedback dos clientes (Zequinão \& Cardoso, 2013), atitude essa que limita a empresa em garantir a fidelização (Larán \& Espinoza, 2004) e a melhorar ou redirecionar os serviços prestados.

Objetivando obter essas informações, (Zequinão \& Cardoso, 2013) desenvolveram um instrumento para avaliar a satisfação de clientes em serviços de Educação Física. Este instrumento, denominado Questionário de Satisfação dos Clientes em Serviços de Educação Física (QSCSEF), possui clareza e validade para mensurar a qualidade percebida, expectativa do cliente antes da utilização do serviço bem como a ocorrência de problemas, porém ainda não foi aplicado em outras regiões.

Frente a isso, o presente estudo objetivou avaliar a satisfação de clientes de serviços em Educação Física supervisionada em Curitiba e Região Metropolitana bem como verificar o efeito do sexo e variáveis socioeconômicas nas dimensões do instrumento.

\section{MÉTODO}

O presente estudo caracteriza-se como uma pesquisa exploratória-descritiva.

\section{Amostra}

A amostra deste estudo refere-se aos clientes de serviços em Educação Física supervisionada, residentes em Curitiba ou Região Metropolitana. Os locais foram selecionados por conveniência e as modalidades avaliadas correspondem àquelas 
enquadradas no instrumento. O número total de participantes, selecionados por conveniência, foi de 921 indivíduos (436 do sexo masculino e 485 do sexo feminino), com idades variando entre 14 e 84 anos e média de $26.9 \pm 9.4$ anos. Todos os procedimentos foram realizados respeitando os princípios éticos da convenção de Helsinque. Todos os participantes assinaram o Termo de Consentimento Livre e Esclarecido e os menores de idade, tiveram este termo assinado por seus pais ou responsáveis e assinaram o Termo de consentimento.

\section{Instrumentos}

$\mathrm{O}$ instrumento de pesquisa selecionado foi o Questionário de Satisfação dos Clientes em Serviços de Educação Física (QSCSEF), criado e validado quanto ao seu conteúdo em um estudo específico de qualidades psicométricas por Zequinão e Cardoso (2013). Neste estudo o QSCSEF foi avaliado quanto ao conteúdo teórico, conteúdo empírico (clareza) e conteúdo analítico (confiabilidade e validade). A avaliação teórica deu-se pela técnica de Delphi e envolveu cinco doutores que analisaram o conteúdo e abrangência do instrumento. A avaliação de clareza contou com a participação de 167 indivíduos, que deram notas de 0 a 10 com relação ao entendimento do QSCSEF e por fim a avaliação analítica, com 194 participantes que responderam o instrumento para análise da confiabilidade (Zequinão \& Cardoso, 2013).

$O$ instrumento de pesquisa é dividido em três dimensões. A primeira é composta por sete questões de ordem social, a fim de caracterizar os participantes quanto às diferenças socioeconômicas. A segunda identifica quais os serviços em Educação Física já utilizados pelo indivíduo, bem como o tempo de utilização dos mesmos. Dentre os serviços avaliados, o questionário apresenta nove opções, sendo uma delas denominada Outros. Neste estudo tal categoria foi preenchida pelos clientes unicamente por corrida ou caminhada. Desta forma, adotou-se Corrida/caminhada como a nona opção de serviço utilizado.

A terceira dimensão é composta por doze questões objetivas e uma questão discursiva. As questões objetivas possuem como resposta uma escala de Likert de 11 pontos (de 0 a 10) onde o indivíduo deve assinalar uma única opção. As primeiras três questões referem-se à expectativa do cliente antes da utilização do serviço. As perguntas de quatro a seis tratam da qualidade percebida após o uso. A pergunta sete era referente ao valor percebido; às perguntas de oito à dez relativas à satisfação. A pergunta onze abordou as reclamações e a pergunta doze a lealdade dos clientes. Por fim, a pergunta discursiva procura verificar quais os tipos de problemas enfrentados pelos clientes (Zequinão \& Cardoso, 2013). Para obtenção do resultado da satisfação foi calculada a pontuação média das doze questões.

\section{Procedimentos}

Os questionários foram aplicados por entrevistadores treinados, cientes do conteúdo e capazes de sanar eventuais dúvidas dos respondentes. Os participantes foram abordados nas em locais públicos (ruas, parques e praças) e convidados a participar do estudo. Aqueles que aceitaram realizaram o preenchimento do questionário, tendo duração aproximada de 10 minutos.

Foram incluídos neste estudo os indivíduos que usufruíram de serviços de Educação Física supervisionada com frequência mínima de duas vezes semanais e que aceitaram preencher o instrumento de pesquisa. Os indivíduos que não preencheram o instrumento em sua totalidade ou que preencheram incorretamente foram excluídos.

\section{Análise estatística}

Após a realização das entrevistas os dados foram tabulados no software Microsoft Office Excel 2010 e estatisticamente analisados pelo software Statistical Package for Social Sciences 21.0. Para caracterização da amostra utilizou-se tabelas de frequência bem como medidas de tendência central (média) e dispersão (desvio padrão). Foi utilizado o teste de KolmogorovSmirnov para verificar a distribuição (normalidade) das variáveis e o teste de KruskalWallis para analisar diferenças entre grupos. Todas as estatísticas foram analisadas considerando um $\mathrm{p}<0.05$ (Maroco, 2007). 


\section{RESULTADOS}

Do Fizeram parte deste estudo 921 participantes, sendo 436 do sexo masculino e 485 do sexo feminino. A média de idade foi de $26.9 \pm$
9.4 anos com idades variando entre 14 a 84 anos. A Tabela 1 descreve as características da amostra quanto aos aspectos sociais e econômicos.

Tabela 1.

Caracterização socioeconômica, por sexo, dos praticantes de atividade física supervisionada em Curitiba e região metropolitana, Paraná, Brasil, 2014.

\begin{tabular}{|c|c|c|c|c|c|c|}
\hline & \multicolumn{2}{|c|}{ Masculino } & \multicolumn{2}{|c|}{ Feminino } & \multicolumn{2}{|c|}{ Todos } \\
\hline & $\mathrm{N}$ & $\%$ & $\mathrm{~N}$ & $\%$ & $\mathrm{~N}$ & $\%$ \\
\hline Sexo & 436 & 47.3 & 485 & 52.7 & 921 & 100 \\
\hline \multicolumn{7}{|l|}{ Faixa Etária } \\
\hline Até 20 Anos & 109 & 25.0 & 111 & 22.9 & 220 & 23.9 \\
\hline 20 a 29 Anos & 212 & 48.6 & 208 & 42.9 & 420 & 45.6 \\
\hline 30 a 39 Anos & 74 & 17.0 & 95 & 19.6 & 169 & 18.3 \\
\hline 40 a 49 Anos & 32 & 7.3 & 57 & 11.8 & 89 & 9.7 \\
\hline 50 a 59 Anos & 9 & 2.1 & 11 & 2.3 & 20 & 2.2 \\
\hline Acima de 60 Anos & 0 & 0.0 & 3 & 0.6 & 3 & 0.3 \\
\hline \multicolumn{7}{|l|}{ Orientação Sexual } \\
\hline Heterossexual & 406 & 93.1 & 463 & 9.5 & 869 & 94.4 \\
\hline Bissexual & 5 & 1.1 & 15 & 3.1 & 20 & 2.2 \\
\hline Homossexual & 25 & 5.7 & 7 & 1.4 & 32 & 3.5 \\
\hline \multicolumn{7}{|l|}{ Reside } \\
\hline Sozinho & 49 & 11.2 & 29 & 6 & 78 & 8.5 \\
\hline Pais & 187 & 42.9 & 178 & 3.7 & 365 & 39.6 \\
\hline Familiares & 123 & 28.2 & 158 & 3.6 & 281 & 30.5 \\
\hline Amigos & 13 & 3 & 11 & 2.3 & 24 & 2.6 \\
\hline Parceiro(a) & 64 & 14.7 & 107 & 22.1 & 171 & 18.6 \\
\hline \multicolumn{7}{|l|}{ Classe econômica } \\
\hline E & 6 & 1.4 & 9 & 1.9 & 15 & 1.6 \\
\hline $\mathrm{D}$ & 22 & 5.0 & 33 & 6.8 & 55 & 6.0 \\
\hline $\mathrm{C}$ & 55 & 12.6 & 63 & 13.0 & 118 & 12.8 \\
\hline B2 & 124 & 28.4 & 131 & 27.0 & 255 & 27.7 \\
\hline B1 & 138 & 31.7 & 149 & 30.7 & 287 & 31.2 \\
\hline A2 & 67 & 15.4 & 84 & 17.3 & 151 & 16.4 \\
\hline A1 & 24 & 5.5 & 16 & 3.3 & 40 & 4.3 \\
\hline \multicolumn{7}{|l|}{ Escolaridade Chefe da Família } \\
\hline Analfabeto/Até $3^{\text {a }}$ Fundamental & 6 & 1.4 & 1 & 0.2 & 7 & 0.8 \\
\hline Até $4^{\mathrm{a}}$ Fundamental & 38 & 8.7 & 46 & 9.5 & 16 & 1.7 \\
\hline Fundamental completo/Médio Incompleto & 70 & 16.1 & 82 & 16.9 & 137 & 14.9 \\
\hline Médio completo/Superior Incompleto & 220 & 50.5 & 240 & 49.5 & 583 & 63.3 \\
\hline Superior completo/Pós-graduação & 102 & 23.4 & 116 & 23.9 & 176 & 19.1 \\
\hline \multicolumn{7}{|l|}{ Escolaridade Respondente } \\
\hline Analfabeto/Até $3^{\text {a }}$ Fundamental & 6 & 1.4 & 1 & 0.2 & 7 & 0.8 \\
\hline Até $4^{\mathrm{a}}$ Fundamental & 4 & 0.9 & 12 & 2.5 & 16 & 1.7 \\
\hline Fundamental completo/Médio Incompleto & 58 & 13.3 & 79 & 16.3 & 137 & 14.9 \\
\hline Médio completo/Superior Incompleto & 290 & 66.5 & 294 & 60.6 & 583 & 63.3 \\
\hline Superior completo/Pós-graduação & 78 & 17.9 & 99 & 20.4 & 176 & 19.1 \\
\hline
\end{tabular}

Com relação a faixas etárias, o maior percentual de usuários de serviços de Educação Física encontra-se entre os 20 a 29 anos (45.6\%) precedidos pela faixa até 20 anos $(23.9 \%)$. Em sua maioria, $70.1 \%$ da amostra residem com parentes, sendo $39.6 \%$ com os pais e $30.5 \%$ com outros familiares.

A maior parte da amostra (58.8\%) é pertencente à classe $\mathrm{B}$, e quanto à escolaridade tanto os chefes de família quanto os respondentes concluíram em sua maioria $\mathrm{O}$ ensino médio (63.3\%). A tabela 2 apresenta os valores de frequência para os serviços em Educação Física utilizados pelos respondentes.

O serviço mais utilizado, para ambos os sexos, foram as academias de musculação ( $64.2 \%$ no masculino; $59.8 \%$ no feminino). Nota-se que o segundo serviço mais utilizado no público em 
geral são as academias de lutas (12.2\%), porém essa característica difere quando analisados os sexos, sendo as academias de lutas a modalidade do público masculino (17.7\%) e as academias de ginástica e academias de dança as modalidades do público feminino (10.3\% e $9.7 \%)$.

O tempo médio de utilização dos serviços foi de $25.2 \pm 33.1$ meses. A tabela 3 mostra a média de tempo de utilização de cada serviço.

Tabela 2

Serviços em Educação Física utilizados pelos praticantes de atividade física supervisionada em Curitiba e região metropolitana, Paraná, Brasil, 2014.

\begin{tabular}{ccccccc}
\hline & \multicolumn{2}{c}{ Masculino } & \multicolumn{2}{c}{ Feminino } & \multicolumn{2}{c}{ Todos } \\
Serviço & $\mathrm{N}$ & $\%$ & $\mathrm{~N}$ & $\%$ & $\mathrm{~N}$ & $\%$ \\
\hline Academias de Musculação & 280 & 64.2 & 290 & 59.8 & 570 & 61.9 \\
Academias de Dança & 20 & 4.6 & 47 & 9.7 & 67 & 7.3 \\
Academias de Ginástica & 5 & 1.1 & 50 & 10.3 & 55 & 6.0 \\
Academias de Yôga/Pilates & 3 & 0.7 & 11 & 2.3 & 14 & 1.5 \\
Academias de Natação/Esportes aquáticos & 24 & 5.5 & 25 & 5.2 & 49 & 5.3 \\
Academias de Lutas & 77 & 17.7 & 34 & 7.0 & 111 & 12.1 \\
Escolas de Esportes Coletivos & 18 & 4.1 & 19 & 3.9 & 37 & 4.0 \\
Espaços Públicos com acompanhamento & 5 & 1.1 & 9 & 1.9 & 14 & 1.5 \\
Corrida/Caminhada & 4 & 0.9 & 0 & 0.0 & 4 & 0.4 \\
\hline
\end{tabular}

Tabela 3

Tempo médio de utilização dos serviços pelos praticantes de atividade física supervisionada em Curitiba e região metropolitana, Paraná, Brasil, 2014.

\begin{tabular}{ccccccc}
\hline & \multicolumn{2}{c}{ Masculino } & \multicolumn{2}{c}{ Feminino } & \multicolumn{2}{c}{ Todos } \\
Serviço & Média & DP & Média & DP & Média & DP \\
\hline Academias de Musculação & 24.9 & 32.4 & 19.6 & 27.5 & 22.2 & 30.1 \\
Academias de Dança & 16.4 & 14.6 & 24.6 & 24.6 & 22.3 & 22.4 \\
Academias de Ginástica & 22.4 & 11.5 & 24.0 & 28.9 & 23.9 & 28.2 \\
Academias de Yôga/Pilates & 5.3 & 3.1 & 15.3 & 19.7 & 13.1 & 17.8 \\
Academias de Natação/Esportes aquáticos & 32.3 & 32.2 & 20.5 & 23.3 & 26.2 & 28.4 \\
Academias de Lutas & 39.7 & 50.2 & 28.5 & 40.4 & 36.2 & 47.5 \\
Escolas de Esportes Coletivos & 39.1 & 32.6 & 54.1 & 53.9 & 48.7 & 45.8 \\
Espaços Públicos com acompanhamento & 25.2 & 24.1 & 19.0 & 17.9 & 21.2 & 19.7 \\
Corrida/Caminhada & 30.0 & 31.4 & 0.0 & 0.0 & 30.0 & 31.4 \\
TOTAL & 28.2 & 36.0 & 22.5 & 30.1 & 25.2 & 33.1 \\
\hline
\end{tabular}

Tabela 4.

Distribuição dos praticantes de atividade física supervisionada em Curitiba e região metropolitana, por classe socioeconômica, Paraná, Brasil, 2014.

\begin{tabular}{|c|c|c|c|c|c|c|c|c|}
\hline \multirow{3}{*}{ Serviço } & \multicolumn{8}{|c|}{ Classe Socioeconômica } \\
\hline & \multicolumn{2}{|c|}{ E e D } & \multicolumn{2}{|c|}{$\mathrm{C}$} & \multicolumn{2}{|c|}{ B } & \multicolumn{2}{|c|}{$\mathrm{A}$} \\
\hline & $\mathrm{N}$ & $\%$ & $\mathrm{n}$ & $\%$ & $\mathrm{n}$ & $\%$ & $\mathrm{n}$ & $\%$ \\
\hline Academias de Musculação & 51 & 72.9 & 79 & 66.9 & 322 & 59.4 & 119 & 62.3 \\
\hline Academias de Dança & 5 & 7.1 & 10 & 8.5 & 38 & 7.0 & 13 & 6.8 \\
\hline Academias de Ginástica & 2 & 2.9 & 4 & 3.4 & 35 & 6.5 & 12 & 6.3 \\
\hline Academias de Yôga/Pilates & 0 & 0.0 & 2 & 1.7 & 5 & 0.9 & 7 & 3.7 \\
\hline Academias de Natação/Esportes aquáticos & 4 & 5.7 & 3 & 2.5 & 36 & 6.6 & 6 & 3.1 \\
\hline Academias de Lutas & 5 & 7.1 & 16 & 13.6 & 67 & 12.4 & 24 & 12.6 \\
\hline Escolas de Esportes Coletivos & 0 & 0.0 & 4 & 3.4 & 24 & 4.4 & 10 & 5.2 \\
\hline Espaços Públicos com acompanhamento & 2 & 2.9 & 0 & 0.0 & 11 & 2.0 & 1 & 0.5 \\
\hline Corrida/Caminhada & 1 & 1.4 & 0 & 0.0 & 2 & 0.4 & 1 & 0.5 \\
\hline
\end{tabular}

As academias de musculação se caracterizaram como o serviço com maior número de clientes, porém na análise de tempo médio de utilização elas ocupam a sétima posição. Os serviços com maior fidelização foram as escolas de esportes coletivos (48.7 meses) e as academias de lutas (36.2 meses) enquanto que as academias de Yôga/Pilates apresentaram a menor média de tempo de utilização (13.1 meses).

A descrição por classes sociais é apresentada na Tabela 4. Novamente percebe-se que as academias de musculação se apresentam como o 
serviço mais utilizado. Um ponto interessante é que os serviços ofertados em locais públicos, criados com o objetivo de ser uma opção para a realização de atividades físicas para pessoas de qualquer classe social, na amostra deste estudo, foram mais utilizados por integrantes da classe B.

O serviço de Yoga/Pilates apresentou seu maior índice entre integrantes da classe $\mathrm{A}$, seguidos pelos pertencentes a classe $\mathrm{B}$, e isso pode ser explicado por ser um serviço mais caro, logo sendo mais utilizado pelas classes mais altas.

A análise de satisfação dos clientes com os serviços de Educação Física deu-se pelas respostas de 12 questões específicas e a questão objetiva. A Tabela 5 apresenta os valores de média (X) e desvio padrão (d) de cada pergunta em cada modalidade e a média geral.

Tabela 5.

Média (X) e Desvio Padrão (DP) da pontuação das questôes do QSCSEF por serviço de atividade física supervisionada em Curitiba e região metropolitana, Paraná, Brasil, 2014.

\begin{tabular}{|c|c|c|c|c|c|c|c|c|c|c|c|c|c|c|}
\hline \multicolumn{2}{|l|}{ Serviço Utilizado } & Q1 & Q2 & Q3 & Q4 & Q5 & Q6 & Q7 & Q8 & Q9 & Q10 & Q11 & Q12 & Total \\
\hline Academias de & $\mathrm{X}$ & 8.1 & 8.3 & 7.6 & 7.9 & 7.9 & 8.1 & 7.9 & 7.9 & 7.5 & 7.4 & 7.9 & 7.7 & 7.8 \\
\hline Musculação & DP & 1.9 & 1.8 & 3.1 & 1.8 & 1.9 & 2.5 & 2.1 & 1.9 & 2.0 & 2.2 & 2.5 & 2.8 & 0.4 \\
\hline Academias de & $\mathrm{X}$ & 8.7 & 8.5 & 7.6 & 8.7 & 8.5 & 8.6 & 8.9 & 9.1 & 8.4 & 8.5 & 8.4 & 8.3 & 8.5 \\
\hline Dança & DP & 1.6 & 1.8 & 3.1 & 1.5 & 1.5 & 2.1 & 1.5 & 1.3 & 1.7 & 1.6 & 2.4 & 2.9 & 0.6 \\
\hline Academias de & $\mathrm{X}$ & 7.5 & 8.6 & 8.5 & 8.1 & 7.7 & 8.2 & 7.8 & 8.1 & 7.6 & 7.5 & 8.3 & 8.1 & 8.0 \\
\hline Ginástica & $\mathrm{DP}$ & 2.8 & 1.6 & 2.1 & 1.7 & 1.6 & 2.1 & 1.8 & 1.6 & 1.6 & 2.0 & 1.5 & 2.5 & 0.4 \\
\hline Academias de & $\mathrm{X}$ & 7.5 & 8.3 & 6.6 & 8.0 & 8.1 & 7.2 & 8.2 & 8.2 & 7.5 & 7.9 & 8.3 & 7.4 & 7.8 \\
\hline Yôga/Pilates & DP & 2.1 & 1.8 & 3.9 & 1.5 & 1.5 & 3.5 & 2.0 & 2.0 & 2.0 & 1.9 & 2.3 & 3.2 & 0.8 \\
\hline Academias de & $\mathrm{X}$ & 8.2 & 8.2 & 8.1 & 8.4 & 8.1 & 8.2 & 8.3 & 8.5 & 7.8 & 7.8 & 8.2 & 7.9 & 8.1 \\
\hline Natação & DP & 1.6 & 1.5 & 2.3 & 1.6 & 1.8 & 2.5 & 2.0 & 1.7 & 2.1 & 2.1 & 2.1 & 3.0 & 0.4 \\
\hline \multirow{2}{*}{ Academias de Lutas } & $\mathrm{X}$ & 8.2 & 8.4 & 8.0 & 8.4 & 8.1 & 8.3 & 8.1 & 8.6 & 7.8 & 7.8 & 8.2 & 7.4 & 8.1 \\
\hline & $\mathrm{DP}$ & 1.9 & 1.6 & 2.7 & 1.8 & 2.1 & 2.3 & 2.2 & 1.7 & 2.1 & 2.1 & 2.2 & 3.4 & 0.5 \\
\hline \multirow{2}{*}{$\begin{array}{c}\text { Escolas de Esportes } \\
\text { Coletivos }\end{array}$} & $\mathrm{X}$ & 8.2 & 7.8 & 7.5 & 7.9 & 7.8 & 7.6 & 8.0 & 8.2 & 7.5 & 7.4 & 7.6 & 6.2 & 7.6 \\
\hline & $\mathrm{DP}$ & 1.8 & 2.1 & 3.1 & 2.0 & 2.3 & 2.7 & 2.3 & 2.1 & 2.1 & 2.1 & 2.6 & 3.7 & 0.5 \\
\hline \multirow{2}{*}{ Espaços Públicos } & $X$ & 8.4 & 7.6 & 8.1 & 8.3 & 7.9 & 7.6 & 7.9 & 7.6 & 7.3 & 7.1 & 7.7 & 7.0 & 7.7 \\
\hline & DP & 1.8 & 1.9 & 2.4 & 1.7 & 1.7 & 2.7 & 3.1 & 2.6 & 2.5 & 2.2 & 2.3 & 3.8 & 0.6 \\
\hline \multirow{2}{*}{ Corrida/Caminhada } & $\mathrm{X}$ & 6.5 & 6.8 & 7.5 & 8.3 & 7.3 & 7.3 & 8.3 & 8.0 & 8.0 & 7.0 & 7.5 & 3.8 & 7.2 \\
\hline & DP & 1.3 & 1.0 & 2.4 & 0.5 & 0.5 & 1.3 & 1.3 & 1.4 & 0.8 & 1.4 & 1.3 & 4.3 & 1.0 \\
\hline \multirow{2}{*}{ TOTAL } & $\mathrm{X}$ & 8.1 & 8.3 & 7.7 & 8.1 & 8.0 & 8.1 & 8.0 & 8.1 & 7.6 & 7.6 & 8.0 & 7.6 & 7.9 \\
\hline & DP & 1.9 & 1.8 & 2.9 & 1.8 & 1.9 & 2.5 & 2.1 & 1.9 & 2.0 & 2.1 & 2.4 & 3.0 & 0.4 \\
\hline
\end{tabular}

As questões Q1 e Q9 estavam relacionadas à expectativa do cliente quanto à qualidade do serviço, sendo a Q1 direcionada ao antes e a Q9 após o uso. Neste conceito as academias de dança se apresentaram como o serviço que obtinha maior expectativa de qualidade pré-utilização bem como o serviço que apresentou a melhor pontuação quanto ao atendimento destas expectativas. O serviço de corrida/caminhada apresentou a menor expectativa de qualidade préutilização $(X=6.5)$, porém apresentou a maior diferença entre pré e pós, obtendo uma pontuação final de média 8.0.

As questões Q2 e Q5 tratavam das exigências pessoais dos clientes, tais como materiais disponíveis, flexibilidade de horários, atendimento pessoal, ambiente agradável, entre outros. O serviço que apresentava maior exigência pessoal eram as academias de ginástica, porém na avaliação pós, foram as academias de dança que obtiveram a maior média $(X=8.5)$.

As questões Q3, Q6, Q11 e a questão discursiva abordaram a frequência que se esperava ocorrer problemas, a frequência com que ocorreram, as reclamações referentes ao serviço e quais foram os problemas encontrados, respectivamente. Foram apontados problemas por $21.4 \%$ dos respondentes $(n=197)$, acumulando 220 problemas citados. Vale ressaltar que o número de problemas citados é maior que o número de respondentes, pois a pergunta era discursiva e poderiam ser apontados mais de um problema.

O serviço com maior percentual de problemas apontados foram as academias de yôga/pilates onde $35.7 \%$ dos clientes tiveram queixas. Os principais problemas foram os relacionados aos recursos humanos ("falta de preparo dos professores", "mau atendimento"). 
O menor índice foi apontado entre as academias de dança, onde apenas $7.5 \%$ dos clientes apontaram problemas, sendo os principais relacionados à infraestrutura do ambiente ("limpeza insuficiente", "espaço reduzido"). Dos utilizadores das academias de musculação, $21.8 \%$ dos clientes relataram ter encontrado problemas, onde os principais foram relacionados aos recursos humanos ("falta de professores", "falta de preparo dos professores", "mau atendimento"), infraestrutura relacionada aos equipamentos ("falta de equipamentos", "equipamentos velhos", "falta de manutenção" e "mau funcionamento"), e lotação ("academia cheia", "aparelhos ocupados" e "demora no atendimento"). As categorias em que os problemas se enquadravam são apresentadas na tabela 6 .

Tabela 6.

Descrição e frequência dos problemas reportados por praticantes de atividade física supervisionada em Curitiba e região metropolitana, Paraná, Brasil, 2014.

\begin{tabular}{|c|c|c|c|}
\hline Problemas Categorizados & Descrição & $\mathrm{N}$ & $\%$ \\
\hline $\begin{array}{l}\text { Infraestrutura } \\
\text { (equipamentos) }\end{array}$ & $\begin{array}{l}\text { Falta de equipamentos ou materiais, equipamentos velhos, falta de manutenção, mau } \\
\text { funcionamento }\end{array}$ & 41 & 18.6 \\
\hline Infraestrutura (ambiente) & $\begin{array}{c}\text { Limpeza insuficiente, espaço reduzido, qualidade dos vestiários, temperatura } \\
\text { inadequada da piscina }\end{array}$ & 25 & 11.4 \\
\hline Recursos Humanos & $\begin{array}{c}\text { Falta de preparo dos professores, atendimento precário dos funcionários, número } \\
\text { insuficiente de professores }\end{array}$ & 82 & 37.3 \\
\hline $\mathrm{V}$ & Mudanças dos valores sem aviso prévio, formas reduzidas de pagamento & 11 & 5.0 \\
\hline & neia, aparelhos & 30 & 13.6 \\
\hline Avaliação & Atraso na avaliação, desmarcação da avaliação sem aviso prévio & 2 & 0.9 \\
\hline Horários & $\begin{array}{c}\text { Atraso no início das atividades, atrasos frequentes dos professores, pouca } \\
\text { disponibilidade de horários }\end{array}$ & 29 & 13.2 \\
\hline TOTAL & & 220 & 100 \\
\hline
\end{tabular}

Dos problemas citados, o maior percentual (37.3\%) foi com relação aos recursos humanos, seguido pelos problemas de infraestrutura de equipamentos (18.6\%). Entre os participantes que apontaram problemas, a maioria possuía idade entre 20 e 29 anos (45.7\%), do sexo masculino $(51.8 \%)$ e pertencente a classe B (59.3\%).

As questões Q4 e Q8 estão ligadas à qualidade do serviço e satisfação do cliente após a utilização. As pontuações dessas questões indicaram as academias de dança como o serviço de maior qualidade e satisfação enquanto que as academias de musculação e espaços públicos se caracterizaram como os de menor qualidade e satisfação.

As questões Q7, Q10 e Q12 avaliaram a compatibilidade entre o preço e o serviço prestado, a proximidade do serviço prestado em comparação com um serviço ideal e a possibilidade de retorno da atividade no mesmo local, respectivamente. O serviço com melhor relação custo-benefício, que mais se aproximava do ideal e com a maior possibilidade de retorno, foram as academias de dança.
Com base nas pontuações obtidas nas doze perguntas objetivas (Q1 a Q12) e na pergunta discursiva, as academias de dança se caracterizaram como o melhor serviço, obtendo uma média geral de 8.5. O serviço com menor pontuação média foi o de corrida/caminhada, cuja média foi de 7.2. Na comparação da pontuação total entre os serviços através do teste de Kruskal-Wallis não foram encontradas diferenças estatisticamente significativas $(\mathrm{p}=0.251)$.

Foram realizadas comparações para verificação de diferença entre os índices de satisfação de cada serviço segundo sexo, classe econômica, idade e escolaridade dos respondentes. Quanto ao sexo a única diferença ocorreu nas academias de lutas, onde o sexo masculino se mostrou mais satisfeito que o sexo feminino $(\mathrm{p}=0.049)$.

$\mathrm{Na}$ análise das classes econômicas foi encontrada diferença apenas no serviço de Natação/Esportes Aquáticos, onde a classe B se mostrou mais satisfeita que as demais $(\mathrm{p}=0.042)$. Com relação a idade, as Escolas de Esporte Coletivos foram o único serviço que obteve diferença, sendo que a faixa etária dos 20 
aos 29 anos mostrou-se mais satisfeita $(\mathrm{p}=0.016)$. Quanto à escolaridade dos respondentes não foram encontradas diferenças na satisfação dos serviços.

\section{DISCUSSÃO}

Os serviços avaliados apresentaram-se como bons em qualidade e satisfação, sendo as academias de dança as que obtiveram o melhor resultado. Foram encontrados poucos estudos avaliando a qualidade de serviços em Educação Física, o que dificultou a comparação entre os resultados. Um estudo realizado em Curitiba (Rech et al., 2011) que avaliou a satisfação com a prática da atividade física, porém não com o serviço, abordou especificamente a caminhada e as atividades físicas moderadas a vigorosas e apontou que a análise de satisfação em atividade física pode estar diretamente relacionada com a intensidade e tipo de atividade, sendo este um ponto a ser considerado em estudos futuros.

De acordo com as faixas etárias, o maior percentual foi de jovens e adultos, já que $87.8 \%$ da amostra possui entre 14 e 39 anos. Valores próximos também foram encontrados em Campina Grande - PB (Medeiros et al., 2012), onde $70 \%$ dos indivíduos participantes do estudo se encontravam entre 16 e 41 anos. Da mesma forma, o público idoso apresentou o menor percentual, sendo de $0.3 \%$ neste estudo e de $3 \%$ em Campina Grande (Medeiros et al., 2012).

O serviço mais utilizado entre os participantes deste estudo foi o de musculação (61.7\%). Valor elevado também foi encontrado na Espanha, onde $51.4 \%$ da amostra era usuária do fitness (Nuviala et al., 2012) Valores menores foram encontrados em outras cidades brasileiras: $40.0 \%$ entre adultos jovens de Rio Claro (Tahara, Schwartz, \& Silva, 2003), 9.3\% entre adolescentes de São Paulo (Garcia \& Fisberg, 2011 ) e $4.7 \%$ e $1.2 \%$ para homens e mulheres adultos, respectivamente, na cidade de Duque de Caxias (Silva, Sandre-Pereira, \& Salles-Costa, 2011).

Este maior valor encontrado no presente estudo pode ser explicado pelo fato de a região de Curitiba apresentar alta sazonalidade climática (Fermino, Reis, \& Cassou, 2008), o que aumenta a busca por espaços fechados e cobertos. Além disso, o número de academias na região é elevado (CREF9/PR, 2013) e com grande diversidade de valores, facilitando o acesso para praticamente todos os públicos (Menezes, 2013; Meyge \& Costanti, 2011).

Em relação aos problemas apontados pelos clientes, neste estudo foi encontrado que o maior índice ocorreu na área de recursos humanos $(37.3 \%)$, com destaque para a falta de preparo dos professores. Em uma amostra de universitários em situação de sobrepeso e obesidade, $30.0 \%$ apontou que problemas com o profissional foram os responsáveis por abandonar a atividade e $50.0 \%$ relatou não retornar à atividade por falta de preparo dos professores (Neto et al., 2013). Uma vez que o papel do professor é despertar nos alunos uma vontade de se manterem ativos e saudáveis (Tahara \& Filho, 2009) e que o mesmo é a figura central do processo pedagógico (Gomes, Chagas, \& Mascarenhas, 2010), torna-se uma importante preocupação das organizações a manutenção de bons profissionais e o cultivo de relações sadias com os clientes, a fim de manter a proximidade da empresa com o cliente e garantir a lealdade (Ferreira, 2012; Ribeiro, Thiesen, \& Tinoco, 2013).

Dentre os serviços avaliados, as academias de Yoga/Pilates apresentaram o maior índice de problemas apontados. Isso pode ser explicado pelo fato de que essas modalidades são usualmente utilizadas pelas classes sociais de maior poder econômico, que por sua vez são mais exigentes em relação ao serviço que estão usufruindo e que por isso, reclama mais (Menezes, 2013).

$\mathrm{Na}$ comparação entre os diferentes serviços, as academias de dança obtiveram as melhores médias, caracterizando-se como o serviço de maior qualidade e satisfação percebida. $\mathrm{O}$ que pode explicar esse resultado é o fato de que, quando comparado a outros serviços como musculação, natação e ginástica, os espaços que oferecem aulas de dança demandam menor necessidade de material e infraestrutura. Além disso, a maior parte dos professores de dança é composta por ex-bailarinos, o que agrega técnica e experiência no ensino da modalidade (Brasileiro, 2013). 
Esse aspecto também é visto nas academias de lutas, onde os professores só podem ministrar aulas após atingirem determinado grau de graduação interna (Drigo, Neto, Cesana, \& Tojal, 2011; Fett \& Fett, 2009). Dessa forma, os aspectos técnicos intrínsecos à modalidade são mantidos com alta qualidade. Outro destaque com relação à graduação interna é que o tempo de prática possui uma proporcionalidade à graduação alcançada (Rufino \& Darido, 2012). Isso também pôde ser visto neste estudo, onde as academias de lutas obtiveram a segunda maior média de tempo de prática no público geral (36.9 meses) e a maior média no público masculino (39.7 meses). Um aliado das academias de lutas é o avanço e popularização de eventos relacionados a lutas, como o UFC, que incitam os indivíduos (principalmente do sexo masculino) a aderirem a esta modalidade. Outro ponto importante é que as lutas representam para o sexo masculino uma oportunidade de demonstrar sua honra e virilidade, sendo um conteúdo intrínseco de construção da identidade e masculinidade (Gastaldo \& Braga, 2011).

Com relação ao tempo de prática, as escolas de esporte coletivo apresentaram a maior média tanto no público geral quanto no feminino (49.1 e 54.1, respectivamente). Esses valores corroboram com o que é apontado na literatura. Segundo Pires e colaboradores (2012) as modalidades de esportes individuais apresentam maior incidência de abandono que as modalidades coletivas e isso ocorre pelo fato de que nos esportes individuais o sucesso depende exclusivamente do desempenho do atleta e não há suporte social por parte dos companheiros da equipe. Um estudo com atletas de futsal também destacou que entre os principais fatores de motivação para a prática, o fator "jogar em equipe" obteve a maior média, tanto em meninos quanto em meninas (Voser, Hernandez, Ortiz, \& Voser, 2014).

Já na utilização de espaços públicos, este estudo apontou que os mesmos obtiveram uma nota final de 7.7 pontos e um grau elevado de satisfação por $78.6 \%$ dos respondentes, além de um tempo médio de 21.2 meses. Bons resultados também foram encontrados em outros estudos no Brasil. Na cidade de Recife a avaliação de um programa público relatou níveis elevados de satisfação em $72.8 \%$ dos participantes e, média de tempo de 27.5 meses (Hallal et al., 2010). Já em Joinville um serviço público ofertado à terceira idade apresentou a satisfação elevada por 93.4\% dos participantes (Salin, Virtuoso, Nepomuceno, Weiers, \& Mazo, 2013). A diferença entre estes resultados pode ser explicada pela diferença de idade já que neste estudo os utilizadores de espaços públicos apresentaram média de idade de $36.4 \pm 18.4$ anos, enquanto que no estudo em Joinville a média de idade foi de $67.2 \pm 5.9$ anos.

Um ponto curioso é que Curitiba é conhecida por apresentar tanto uma boa gama de espaços públicos destinados à pratica de atividade física, quanto políticas públicas de incentivo à promoção de saúde (Hallal, Reis, \& Hino, 2009), porém isto não pode ser visto neste estudo, onde a utilização dos espaços públicos foi apontada por apenas $1.5 \%$ da amostra.

A análise da satisfação apresentou pouca diferença entre os serviços analisados, sendo encontradas maiores índices de satisfação apenas nas academias de lutas para o sexo masculino $(\mathrm{p}=0.049)$; nas academias de natação $\mathrm{e}$ atividades aquáticas para a classe $B(p=0.042)$; e nas escolas de esportes coletivos para a faixa etária de 20 a 29 anos $(\mathrm{p}=0.016)$. Um estudo realizado na Espanha comparou serviços de fitness, natação e esportes de raquete, porém não encontrou diferença entre os níveis de satisfação dos praticantes. Não foram encontrados estudos brasileiros utilizando o mesmo instrumento ou que realizaram comparação semelhante à feita neste estudo.

O QSCSEF apresentou-se como um instrumento adequado para avaliação da satisfação dos clientes, porém foram observadas limitações neste estudo. O primeiro ponto é que o instrumento não apresenta de forma direta uma nota final sobre o serviço avaliado, sendo este resultado obtido apenas quando realizada a soma das doze pontuações das perguntas objetivas. Além disso, ressalta-se que a pergunta discursiva não é computada na soma do resultado final, pois se apresenta qualitativamente, necessitando de uma forma de pontuação quantitativa para somar-se às perguntas objetivas. Por se tratar de 
uma pergunta aberta, as respostas por vezes são amplas e generalistas, dificultando a especificação detalhada dos problemas apontados pelos respondentes. Outro ponto a ser considerado é que as opções de serviços incluídas no QSCSEF por vezes não são encontradas no mercado, uma vez que muitas academias oferecem diversos serviços como ginástica, dança, natação, hidroginástica, musculação, esportes e tênis (Mello \& Silva, 2013; Menezes, 2013), o que dificulta no momento de avaliação, sendo este um ponto que necessita de atenção quando da utilização do instrumento.

\section{CONCLUSÕES}

Os serviços em Educação Física analisados neste estudo se apresentaram de forma geral como bons em qualidade e satisfação dos clientes. Dentre os serviços avaliados, as academias de musculação se caracterizaram como o mais utilizado, sendo a satisfação significativamente maior para o sexo masculino.

Com relação aos problemas evidenciados, as academias de yôga/pilates obtiveram o maior percentual de reclamações, sendo a maior parte relacionada aos profissionais de Educação Física. Esta problemática pode assumir diversas hipóteses, indo desde a formação acadêmica do profissional até seu engajamento no mercado de trabalho, sendo este um ponto que merece atenção, pois influi diretamente na satisfação e fidelidade dos clientes. No aspecto da qualidade e satisfação, as academias de dança apresentaram o maior índice, e o serviço de caminhada/corrida o menor.

\section{Agradecimentos:}

LAERG - UTFPR

\section{Conflito de Interesses:}

Nada a declarar.

Financiamento:

Nada a declarar

\section{REFERÊNCIAS}

Batista, M. da G., Couto, J. P., Botelho, D., \& Faias, C. (2014). Tourist satisfaction and loyalty in the hotel business: An application to the island of
São Miguel, Azores. Tourism \& Management Studies, 10(1), 16-23.

Borrego, C. M. C., Leitão, J. C., Alves, J., Silva, C., \& Palmi, J. (2000). Análise Confirmatória do Questionário de Satisfação do Atleta - Versão Portuguesa. Psicologia: Reflexão E Crítica, 23(1), 110-120.

Brasileiro, L. T. (2013). Na dança tanto seu objeto quanto seu instrumento profissional é o próprio corpo. Revista Brasileira de Ciências Do Esporte, 35(2), 311-326.

Costa, H. G., Mansur, A. F. U., Freitas, A. L. P., \& Carvalho, R. A. de. (2007). ELECTRE TRI aplicado a avaliação da satisfação de consumidores. Revista Produção, 17(2), 230245.

CREF9/PR. (2013). CREF9/PR promove $1^{\circ}$ Encontro de Empresários de Academias e Atividades Físicas, em parceria com IBQP e CONFEF. Retrieved September 10, 2014, from http://www.crefpr.org.br/?p=4594

Drigo, A. J., Neto, S. de S., Cesana, J., \& Tojal, J. B. A. G. (2011). Artes marciais, formação profissional e escolas de ofício: Análise documental do judô brasileiro. Motricidade, 7(4), 49-62.

Fermino, R. C., Reis, R. S., \& Cassou, A. C. (2008). Fatores individuais e ambientais associados ao uso de parques e praças por adultos de CuritibaPR, Brasil. Revista Brasileira de Cineantropometria E Desempenho Humano, 14(4), 377-389.

Fernandes, P. O. (2013). Atitudes do consumidor em relação às práticas do marketing em Portugal. Tourism \& Management Studies, 9(2), 86-92.

Ferreira, C. M. C. (2012). Um estudo sobre fidelização e retenção de clientes na área do fitness. Insituto Politécnico de Castelo Branco.

Fett, C. A., \& Fett, W. C. R. (2009). Filosofia , ciência e a formação do profissional de artes marciais Artes marciais e saúde. Motriz, 15(1), 173-184.

Gabriel, M. O., Pelissari, A. S., \& Oliveira, M. P. V. de. (2014). Relacionamento com clientes do setor farmacêutico em vitória-es: uma análise dos impactos do nível de serviço esperado e ofertado na fidelização dos clientes. Revista Eletrônica de Administração, 77(1), 64-89.

Garcia, L., \& Fisberg, M. (2011). Atividades físicas e barreiras referidas por adolescentes atendidos num serviço de saúde. DOI: 10.5007/19800037.2011v13n3p163. Revista Brasileira de Cineantropometria E Desempenho Humano, 13(3), 163-169. doi:10.5007/19800037.2011v13n3p163

Gastaldo, É. L., \& Braga, A. A. (2011). Corporeidade, esporte e identidade masculina. Estudos Feministas, 19(3), 875-893.

Gomes, A. R., Pereira, A. P., \& Pinheiro, A. R. (2008). Liderança , Coesão e Satisfação em Equipas Desportivas: Um Estudo com Atletas Portugueses de Futebol e Futsal. Psicologia: Reflexão E Crítica, 21(3), 482-491. 
Gomes, I. R., Chagas, R. de A., \& Mascarenhas, F. (2010). A indústria do Fitness , a mercantilização das práticas corporais e o trabalho do professor de Educação Física: o caso Body Systems. Movimento, 16(4), 169-189.

Hallal, P. C., Reis, R. S., \& Hino, A. A. F. (2009). Avaliação de Programas Comunitários de Promoção da Atividade Física : o caso de Curitiba, Paraná. Revista Brasileira de Atividade Física E Saúde, 14(2), 104-114.

Hallal, P. C., Tenório, M. C. M., Tassitano, R. M., Reis, R. S., Carvalho, Y. M., Cruz, D. K. A., ... Malta, D. C. (2010). Avaliação do programa de promoção da atividade física Academia da Cidade de Recife, Pernambuco, Brasil: percepções de usuários e não-usuários. Caderno de Saúde Pública, 26(1), 70-78.

Larán, J. A., \& Espinoza, F. da S. (2004). Consumidores Satisfeitos, e Então? Analisando a Satisfação como Antecedente da Lealdade. Revista de Administração Contemporânea, 8(2), 51-70.

Maroco, J. (2007). Statistical Analysis with SPSS application. Lisboa: Edições Silabo.

Medeiros, F. G., Andrade, R. D. S., Oliveira, T. D. A., Júnior, A. T. de A., \& Sousa, M. do S. C. de. (2012). Gestão empresarial em academias de ginástica: relação cliente e utilização dos serviços na cidade Campina Grande - Paraíba. Coleção Pesquisa Em Educação Física, 11(4), 111-118.

Mello, J. A. C. de, \& Silva, S. A. P. dos S. (2013). Competências do gestor de academias esportivas. Revista Motriz, 19(1), 74-83.

Menezes, R. da C. (2013). O Forte do mercado Um análise do mercado de fitness não convencional. Fundação Getúlio Vargas Escola Brasileira de Administração Pública e de Empresas.

Meyge, A., \& Costanti, S. (2011). Consumidor de baixa renda já frequenta academia de ginástica. Retrieved September 10, 2014, from http://www.educacaofisica.com.br/index.php/g estao/canais-gestao/academias/18151consumidor-de-baixa-renda-ja-frequentaacademia-de-ginastica

Neto, J. B., Pinto, J. S., Silva, J. V. P., Sales, M. M., Córdova, C., Pereira, L. A., \& Asano, R. Y. (2013). Motivos do abandono de academias de ginástica por universitários com sobrepeso ou obesidade. Revista Brasileira Ciência E Movimento, 21(3), 96-104.

Nuviala, A. N., Pérez-Ordás, R., Osuna, M. B., GraoCruces, A., Nuviala, R. N., \& Jurado, J. A. G. (2012). Calidad, satisfacción y valor percibido de los usuarios de un servicio desportivo público. Movimento, 18(4), 11-32.

Pires, D. A., Santiago, M. L. D. M., Samulski, D. M., \& Costa, V. T. Da. (2012). A Síndrome de Burnout no Esporte Brasileiro. Revista Da Educação Física/UEM, 23(1), 131-139. doi:10.4025/reveducfis.v23i1.14566

Rech, C. R., Fermino, R. C., Hallal, P. C., \& Reis, R. S. (2011). Validade e fidedignidade da escala de satisfação com a prática de atividade física em adultos. Revista de Saúde Pública, 45(2), 286293.

Ribeiro, J. L. D., Thiesen, J. P. K., \& Tinoco, M. A. C. (2013). Determinantes da satisfação e atributos da qualidade em serviços de salão de beleza. Revista Produção, 23(3), 609-624.

Rufino, L. G. B., \& Darido, S. C. (2012). Pedagogia do esporte e das lutas: em busca de aproximações. Revista Brasileira de Educação Física E Esporte, 26(2), 283-300.

Saldanha, L. C. (2013). Gestão da Produção: Integração Entre o Planejamento e Controle da produção e a Gestão da Qualidade. Universidade Federal do Rio Grande do Sul.

Salin, M. da S., Virtuoso, J. F., Nepomuceno, A. S. N., Weiers, G. G., \& Mazo, G. Z. (2013). Golden Age Gym: reasons for entry , permanence and satisfaction among participating older adults. Revista Brasileira de Cineantropometria $E$ Desempenho Humano, 16(2), 152-160.

Silva, S. P. de S., Sandre-Pereira, G., \& Salles-Costa, R. (2011). Fatores sociodemográficos e atividade física de lazer entre homens e mulheres de Duque de Caxias / RJ. Ciência E Saúde Coletiva, 16(11), 4493-4501.

Tahara, A. K., \& Filho, S. C. (2009). Atividades físicas de aventura na natureza (AFAN) e academias de ginástica: motivos de aderência e benefícios advindos da prática. Movimento, 15(3), 187-208.

Tahara, A. K., Schwartz, G. M., \& Silva, K. A. (2003). Aderência e manutenção da prática de exercícios em academias. Revista Brasileira Ciência $E$ Movimento, 11(4), 7-11.

Voser, R. da C., Hernandez, J. A. E., Ortiz, L. F. R., \& Voser, P. E. G. (2014). A motivação para a prática do futsal: comparação entre atletas federados do sexo masculino e feminino. Revista Brasileira de Futsal E Futebol, 6(21), 196-201.

Zequinão, M. A., \& Cardoso, F. L. (2013). Criação e avaliação das qualidades psicométricas do questionário de satisfação dos clientes em serviços de Educação física (QSCSEF). Revista Brasileira de Ciências Do Esporte, 35(2), 481501. 